
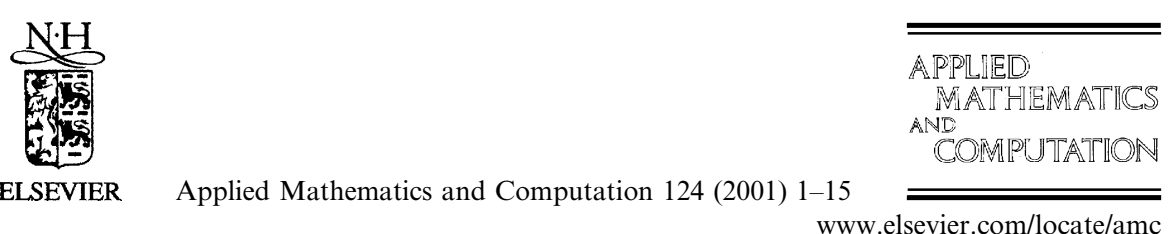

www.elsevier.com/locate/amc

\title{
Bayesian analysis for detecting a change in exponential family
}

\author{
Dariush Ghorbanzadeh ${ }^{\mathrm{a}, *}$, Rachid Lounes ${ }^{\mathrm{b}}$ \\ a Département Mathématiques, Conservat. Nat. des Arts et Metiers, 292 Rue Saint-Martin, \\ 75141 Paris Cedex 03, France \\ ${ }^{\mathrm{b}}$ Laboratoire de Statistique Médicale, Université René Descartes, UA CNRS 1323, \\ 45 Rue des Saints-Pères, 75006 Paris Cedex, France
}

\begin{abstract}
We propose a Bayesian analysis of detection of a change of parameter in a sequence of independent random variables from exponential family. The test uses the highest posterior density credible set. (C) 2001 Elsevier Science Inc. All rights reserved.
\end{abstract}

Keywords: Change-point; Posterior distribution; HPD credible set; $p$-Value

\section{Introduction}

We consider a sequence $X_{1}, \ldots, X_{n}$ of independent random variables such that there exists $k \in\{1, \ldots, n\}$ so that $X_{1}, \ldots, X_{k}$ have a distribution $F_{\theta_{1}}$ and $X_{k+1}, \ldots, X_{n}$ have a distribution $F_{\theta_{2}}$.

We suppose that $\theta_{1}, \theta_{2}, k$ are unknown and $k$ is the said change-point. The aim of this work is to estimate $k$ from the observed sequence $\left(x_{1}, \ldots, x_{n}\right)$. By the Bayes formula, we determine the posterior distribution of the change-point $k$.

The problem of detection of change in Bayesain context was studied by many authors. We can cite the works of Chernoff and Zacks [1], Kander and Zacks [8] and Sen and Srivatana (1973) where the aim is to detect the change in the mean for normal random variables; Menzefricke [11] proposed a test of

\footnotetext{
${ }^{*}$ Corresponding author.

E-mail addresses: ghorbanzadeh@vcnam.cnam.fr (D. Ghorbanzadeh), lounes@citi2.fr (R. Lounes).
} 
change in the variance for the same family of observations. Smith [12] proposed a Bayesian procedure for both normal and binomial random variables and Kim [9] in linear regression context. Henderson and Matthews [7] proposed a non-Baysian model for Poisson random variables to detect changes in annual number of cases of haemolytic uraemic syndrome (HUS). They studied not only at most one change but also multi-change. West and Ogden [13] used the HUS data for the estimation problem of change-points in a sequence of Poisson random variables approached by allowing the change to range over a continuous time interval $(0, T)$, and derived a Bayesian-based interval estimator. Lee [10] proposed a Bayesian test for an exponential random family based on the Type II maximum likelihood (ML-II) approach. The objectives of these works are different from our work. We place our work in a similar situation as in Ghorbanzadeh and Lounes [4] who proposed a Bayesian test for exponential random variables. They introduced a parameter nuisance as in [3] and confirmed Smith's result, but their confirmation depended on the value of this parameter.

We apply the results obtained to the data in [2], on the annual volume of discharge from the Nile River at the Aswan dam in the period 1871 to 1970 for the exponential case, and for the Poisson case we use the data in [7].

\section{Statistical model and test procedure}

Let $F_{\theta}$ denote an exponential family of the form

$$
\mathrm{d} F_{\theta}(x)=a(x)(\phi(\theta))^{b(x)} \mathrm{e}^{-\psi(\theta) c(x)} \mathrm{d} \mu(x)
$$

relative to some Lebesgue measure $\mu$.

Our model includes the following cases:

A. Continuous cases

1. If $c(x)=x^{2} / 2, b(x)=(1 / 2) a(x)=1 / \sqrt{2 \pi}, \phi(\theta)=\psi(\theta)$, then $X \sim \mathcal{N}(0,1 /$ $\psi(\theta))$.

2. If $a(x)=x^{\alpha-1}, \quad b(x)=\alpha \quad$ (known), $\quad c(x)=x, \quad \phi(\theta)=\psi(\theta), \quad$ then $\quad X \sim$ $\operatorname{Gamma}(\alpha, \psi(\theta))$.

3. If $a(x)=1, b(x)=1, c(x)=|x|, \phi(\theta)=\psi(\theta)$, then $X \sim$ Double-Exponen$\operatorname{tial}(\psi(\theta))$.

B. Discrete cases

1. If $a(x)=1 / x !, b(x)=x, c(x)=1, \phi(\theta)=\psi(\theta)$, then $X \sim$ Poisson $(\psi(\theta))$.

2. If $a(x)=1, b(x)=1-x, c(x)=x, \phi(\theta)=1-\theta, \psi(\theta)=-\log \theta$, then $X \sim$ Bernoulli $(\theta)$.

3. If $a(x)=\left(\begin{array}{c}m \\ x\end{array}\right), b(x)=m-x, c(x)=x, \phi(\theta)=1-\theta, \psi(\theta)=-\log \theta$, then $X \sim \operatorname{Binomial}(m, \theta)$. 
4. If $a(x)=\left(\begin{array}{c}x+r+1 \\ x\end{array}\right), b(x)=x, c(x)=r$ (known), $\phi(\theta)=1-\theta, \psi(\theta)=$ $-\log \theta$, then $X \sim$ Negative-Binomial $(r, \theta)$.

In this work, we consider a changing model

$$
X_{i} \mid \theta_{1}, \theta_{2}, k \sim \begin{cases}F_{\theta_{1}} & \text { if } i=1, \ldots, k, \\ F_{\theta_{2}} & \text { if } i=k+1, \ldots, n .\end{cases}
$$

We suppose that $\theta_{1}, \theta_{2}$ and $k$ are independent.

We build an inference about testing the hypothesis and estimating the change-point $k$. That is, to test whether or not a change-point occurs, i.e.

$$
\mathrm{H}_{0}: \theta_{1}=\theta_{2} \text { against } \mathrm{H}_{1}: \theta_{1} \neq \theta_{2} \text {. }
$$

The proposed test uses Bayesian analysis, based on the posterior distribution of the ratio $\lambda=\theta_{1} / \theta_{2}$. The hypothesis $\mathrm{H}_{0}$ meaning "no change" is equivalent to $\mathrm{H}_{0}^{\prime}: k=n$ and $\mathrm{H}_{1}$ equivalent to $\mathrm{H}_{1}^{\prime}: k \neq n$. The test $\mathrm{H}_{0}^{\prime}$ against $\mathrm{H}_{1}^{\prime}$ is based on the posterior distribution of the change-point $k[3,10]$. The advantage of this procedure is that asymptotics is not required [6], unlike in non-Bayesian classical procedures $[4,5]$.

\subsection{Choice of prior}

To cover many classical distributions, we suppose the following two hypotheses: we impose

1. $\psi(\theta)=\phi(\theta)=\theta \quad$ if $\theta \in] 0,+\infty[$,

2. $\psi(\theta)=-\log \theta, \phi(\theta)=1-\theta$ if $\theta \in] 0,1[$.

We suppose that the parameters $\theta_{j}, j=1,2$, have the Gamma prior distribution: $\pi_{j}\left(\theta_{j}\right) \sim \operatorname{Gamma}\left(\mu_{j}, v_{j}\right)$ for the case $\left.\theta_{j} \in\right] 0,+\infty[$, and the Beta prior distribution: $\pi_{j}\left(\theta_{j}\right) \sim \operatorname{Beta}\left(v_{j}, \mu_{j}\right)$ for the case $\left.\theta_{j} \in\right] 0,1[$ where the parameters, $\mu_{1}, \mu_{2}, v_{1}$ and $v_{2}$ are assumed to be known.

These choices are motivated by technical calculus and are usually used in Bayesian change-point literature [3,6,12].

For the prior of $k$ we use discrete families defined as $\{1, \ldots, n\}$ built on a uniform distribution of $[0,1]$ for a parameter $\theta$ :

$$
\pi(k \mid \theta)= \begin{cases}q_{n}(\theta) & \text { if } k=n, \\ p_{n}(k, \theta) & \text { if } k=1, \ldots, n-1 .\end{cases}
$$

Let $v(k)=\int_{0}^{1} \pi(k \mid \theta) \mathrm{d} \theta$. Then

$$
\begin{aligned}
v(k) & = \begin{cases}\int_{0}^{1} q_{n}(\theta) \mathrm{d} \theta & \text { if } k=n, \\
\int_{0}^{1} p_{n}(k, \theta) \mathrm{d} \theta & \text { if } k=1, \ldots, n-1 .\end{cases} \\
& = \begin{cases}q_{n} & \text { if } k=n, \\
p_{n}(k) & \text { if } k=1, \ldots, n-1 .\end{cases}
\end{aligned}
$$




\subsection{Posterior distribution of $k$}

Let

$$
\begin{aligned}
& b_{k}=\sum_{i=1}^{k} b\left(x_{i}\right), \quad b_{k}^{*}=\sum_{i=k+1}^{n} b\left(x_{i}\right), \\
& c_{k}=\sum_{i=1}^{k} c\left(x_{i}\right), \quad c_{k}^{*}=\sum_{i=k+1}^{n} c\left(x_{i}\right), \quad \text { and } \quad \underline{x}=\left(x_{1}, \ldots, x_{n}\right) .
\end{aligned}
$$

We have

$$
l\left(\underline{x} \mid \theta_{1}, \theta_{2}, k\right)=\left(\prod_{i=1}^{n} a\left(x_{i}\right)\right) \phi\left(\theta_{1}\right)^{b_{k}} \phi\left(\theta_{2}\right)^{b_{k}^{*}} \mathrm{e}^{-c_{k} \psi\left(\theta_{1}\right)-c_{k}^{*} \psi\left(\theta_{2}\right)}
$$

with the convention $b_{n}^{*}=c_{n}^{*}=0$.

By the classical Bayes formula, we obtain

$$
\begin{aligned}
& \pi\left(\underline{x}, \theta_{1}, \theta_{2}, k\right)=l\left(\underline{x} \mid \theta_{1}, \theta_{2}, k\right) \pi\left(\theta_{1}, \theta_{2}\right) v(k), \\
& \pi(\underline{x}, k)=\int_{0}^{\infty} \int_{0}^{\infty} \pi\left(\underline{x}, \theta_{1}, \theta_{2}, k\right) \pi_{1}\left(\theta_{1}\right) \pi_{2}\left(\theta_{2}\right) \mathrm{d} \theta_{1} \mathrm{~d} \theta_{2}
\end{aligned}
$$

and

$$
\pi\left(\theta_{1}, \theta_{2} \mid \underline{x}, k\right)= \begin{cases}\frac{\left(c_{k}+v_{1}\right)^{b_{k}+\mu_{1}}}{\Gamma\left(b_{k}+\mu_{1}\right)} \theta_{1}^{b_{k}+\mu_{1}-1} \mathrm{e}^{-\theta_{1}\left(c_{k}+v_{1}\right)} & \text { if } \theta \in] 0,+\infty[, \\ \times \frac{\left(c_{k}^{*}+v_{2}\right)^{b_{k}^{*}+\mu_{2}}}{\Gamma\left(b_{k}^{*}+\mu_{2}\right)} \theta_{2}^{b_{k}^{*}+\mu_{2}-1} \mathrm{e}^{-\theta_{2}\left(c_{k}^{*}+v_{2}\right)} & \text { if } \theta \in] 0,1[, \\ \frac{\theta_{1}^{c_{k}+v_{1}-1}\left(1-\theta_{1}\right)^{b_{k}+\mu_{1}-1}}{\beta\left(b_{k}+\mu_{1}, c_{k}+v_{1}\right)} \frac{\theta_{2}^{c_{k}^{*}+v_{2}-1}\left(1-\theta_{2}\right)^{b_{k}^{*}+\mu_{2}-1}}{\beta\left(b_{k}^{*}+\mu_{2}, c_{k}^{*}+v_{2}\right)} & \end{cases}
$$

where $\Gamma(\cdot)$ and $\beta(\cdot, \cdot)$ are the gamma and beta functions, respectively.

We can state that, given as $(\underline{x}, k), \theta_{1}$ and $\theta_{2}$ are independent and follow

$$
\begin{aligned}
& \theta_{1} \mid \underline{x}, k \sim \begin{cases}\operatorname{Gamma}\left(b_{k}+\mu_{1}, c_{k}+v_{1}\right) & \text { if } \theta \in] 0,+\infty[, \\
\operatorname{Beta}\left(c_{k}+v_{1}, b_{k}+\mu_{1}\right) & \text { if } \theta \in] 0,1[.\end{cases} \\
& \theta_{2} \mid \underline{x}, k \sim \begin{cases}\operatorname{Gamma}\left(b_{k}^{*}+\mu_{2}, c_{k}^{*}+v_{2}\right) & \text { if } \theta \in] 0,+\infty[, \\
\operatorname{Beta}\left(c_{k}^{*}+v_{2}, b_{k}^{*}+\mu_{2}\right) & \text { if } \theta \in] 0,1[.\end{cases} \\
& \pi(\underline{x}, k)= \begin{cases}v(k) \frac{\Gamma\left(b_{k}+\mu_{1}\right) \Gamma\left(b_{k}^{*}+\mu_{2}\right)}{\left(c_{k}+v_{1}\right)^{b_{k}+\mu_{1}}\left(c_{k}^{*}+v_{2}\right)_{k}^{*}+\mu_{2}} & \text { if } \theta \in] 0,+\infty[, \\
v(k) \beta\left(c_{k}+v_{1}, b_{k}+\mu_{1}\right) \beta\left(c_{k}^{*}+v_{2}, b_{k}^{*}+\mu_{2}\right) & \text { if } \theta \in] 0,1[.\end{cases}
\end{aligned}
$$

Then, the posterior distribution of $k$ is given by $v^{*}(k \mid \underline{x})=\pi(\underline{x}, k) / f(\underline{x})$ with $f(\underline{x})=\sum_{k=1}^{n} \pi(\underline{x}, k)$.

We can remark that $v$ and $v^{*}$ are conjugate. 


\subsection{Statistic Test}

Let $\lambda=\theta_{1} / \theta_{2}$. In this section we suppose that $\left.\theta \in\right] 0,+\infty[$, thus we have

Proposition 1. Given as $(\underline{x}, k)$

$$
\pi^{*}(\lambda \mid \underline{x}, k)=\left(\frac{c_{k}+v_{1}}{c_{k}^{*}+v_{2}}\right)^{b_{k}+\mu_{1}} \frac{1}{\beta\left(b_{k}+\mu_{1}, b_{k}^{*}+\mu_{2}\right)} \frac{\lambda^{b_{k}+\mu_{1}-1}}{\left[1+\frac{c_{k}+v_{1}}{c_{k}^{*}+v_{2}} \lambda\right]^{\mu_{1}+\mu_{2}+b_{n}}} .
$$

By making the following change of variables: $\left(\lambda=\theta_{1} / \theta_{2}, u=\theta_{1}\right)$ we obtain the result.

Let

$$
R_{k}(\lambda)=\left(c_{k}+v_{1}\right) /\left(c_{k}^{*}+v_{2}\right) \lambda \quad \text { and } \quad D_{k}(\lambda)=\left(b_{k}^{*}+\mu_{2}\right) /\left(b_{k}+\mu_{1}\right) R_{k}(\lambda) .
$$

Then we have

Proposition 2. Given as $(\underline{x}, k)$

1. $R_{k}(\lambda) \sim \beta_{I I}\left(b_{k}, b_{k}^{*}\right)$

2. If for all $k$, both $b_{k}+\mu_{1}$ and $b_{k}^{*}+\mu_{2}$ are integers, then $D_{k}(\lambda) \sim F_{2\left(b_{k}+\mu_{1}\right), 2\left(b_{k}^{*}+\mu_{2}\right)}$ where $\beta_{I I}(a, b)$ denotes the Beta distribution of the second kind with density

$$
\frac{1}{\beta(a, b)} \frac{x^{a-1}}{(1+x)^{a+b}} \quad \forall x>0, \quad a>0, \quad b>0 .
$$

If $X \sim \beta_{I I}(a, b)$ (with $a \in \mathbb{N}^{*}, b \in \mathbb{N}^{*}$ ), then $(b / a) X \sim F_{2 a, 2 b}$, where $F_{n_{1}, n_{2}}$ is the Fisher distribution with $\left(n_{1}, n_{2}\right)$ degrees of freedom.

\subsection{Rejection zone}

Given as $k$, a $(1-\alpha)$ credible set for $D_{k}(\lambda)$ is defined by

$$
S_{k}(\alpha)=\left\{D_{k}(\lambda): \mathscr{F}_{2\left(b_{k}+\mu_{1}\right), 2\left(b_{k}^{*}+\mu_{2}\right)}(\alpha / 2) \leqslant D_{k}(\lambda) \leqslant \mathscr{F}_{2\left(b_{k}+\mu_{1}\right), 2\left(b_{k}^{*}+\mu_{2}\right)}(1-\alpha / 2)\right\},
$$

where $\mathscr{F}_{n_{1}, n_{2}}(\alpha)$ is the $\alpha$-quantile of $F_{n_{1}, n_{2}}$.

The decision rule for rejecting $H_{0}$ at the $(100 \alpha) \%$ level is

$$
D_{k}(\lambda=1)=\frac{b_{k}^{*}+\mu_{2}}{b_{k}+\mu_{1}} \frac{c_{k}+v_{1}}{c_{k}^{*}+v_{2}} \notin S_{k}(\alpha) .
$$

Given as $k$, the $p$-value is

$$
p_{\lambda=1}(k)=2 \min \left\{P\left(Y<D_{k}(1) \mid \underline{x}, k\right), P\left(Y>D_{k}(1) \mid \underline{x}, k\right)\right\},
$$


where $Y \sim F_{2\left(b_{k}+\mu_{1}\right), 2\left(b_{k}^{*}+\mu_{2}\right)}$. Then we have

$$
p_{\lambda=1}(k)=2 \min \left\{\mathscr{F}_{2\left(b_{k}+\mu_{1}\right), 2\left(b_{k}^{*}+\mu_{2}\right)}\left(D_{k}(1)\right), 1-\mathscr{F}_{2\left(b_{k}+\mu_{1}\right), 2\left(b_{k}^{*}+\mu_{2}\right)}\left(D_{k}(1)\right)\right\},
$$

where $\mathscr{F}_{2\left(b_{k}+\mu_{1}\right), 2\left(b_{k}^{*}+\mu_{2}\right)}$ is the cumulative distribution function of $F_{2\left(b_{k}+\mu_{1}\right), 2\left(b_{k}^{*}+\mu_{2}\right)}$.

The unconditional $p$-value is

$$
p_{\lambda=1}=\sum_{k=1}^{n} p_{\lambda=1}(k) v^{*}(k \mid \underline{x}) .
$$

\section{Application}

For the parameter $k$, we propose the three following distributions:

$$
\begin{aligned}
& \pi_{1}(k \mid \theta)= \begin{cases}\theta^{n-1} & \text { if } k=n, \\
(1-\theta) \theta^{k-1} & \text { if } 1 \leqslant k \leqslant n-1 .\end{cases} \\
& v_{1}(k)=\left\{\begin{array}{ll}
1 / n & \text { if } k=n, \\
1 / k(k+1) & \text { if } 1 \leqslant k \leqslant n-1 .
\end{array} \text { if } k=n,\right. \\
& \pi_{2}(k \mid \theta)= \begin{cases}\theta & \text { if } 1 \leqslant k \leqslant n-1 . \\
\theta(1-\theta)^{k} / 1-(1-\theta)^{n-1} & \text { if } k=n,\end{cases} \\
& v_{2}(k)= \begin{cases}1 / 2 & \text { if } 1 \leqslant k \leqslant n-1 . \\
\int_{0}^{1} \theta(1-\theta)^{k-1} / 1-(1-\theta)^{n-1} \mathrm{~d} \theta & \text { if } k=n,\end{cases} \\
& \pi_{3}(k \mid \theta)= \begin{cases}\theta & \text { if } 1 \leqslant k \leqslant n-1 . \\
1-\theta / n-1 & \text { if } k=n,\end{cases} \\
& v_{3}(k)= \begin{cases}1 / 2 & \text { if } 1 \leqslant k \leqslant n-1 . \\
1 / 2(n-1) & \end{cases}
\end{aligned}
$$

\subsection{The exponential case}

In this section, we apply the previous results to the data in [2], on the annual volume of discharge from the Nile River at the Aswan dam in the period 1871 to 1970. Cobb [2] supposes that the data come from a normal distribution and shows by a different technique that there is a change in the mean at $k=28$ (year 1898). Since for these data, the change occurs for the mean, and because our model for the normal case is adapted for a change in variance, we transform these data to an exponential one. 
Let

$$
X=-\sigma \log \left(1-\Phi\left(\frac{Y-m}{\sigma}\right)\right)
$$

where $Y \sim \mathscr{N}(m, \sigma)$ and $\Phi$ is the cumulative function of a standard normal random variable; it is easy to show that $X \sim \exp (1 / \sigma)$.

In our application, we have replaced $m$ and $\sigma$ by their empirical estimators.

For the $v_{1}$ prior distribution, we obtain the data presented in Table 1 (see also Fig. 1).

Table 1

The posterior probability $v_{1}^{*}(k \mid \underline{x})$ of the change-point $k$ in Nile data

\begin{tabular}{lllll}
\hline Year & $k$ & $v_{1}^{*}(k \mid \underline{x})$ & $p_{\lambda=1}(k)$ & $v_{1}^{*}(k \mid \underline{x}) p_{\lambda=1}(k)$ \\
\hline 1895 & 25 & $8.1781 \times 10^{-3}$ & $7.3382 \times 10^{-9}$ & $6.0012 \times 10^{-11}$ \\
1896 & 26 & $1.0605 \times 10^{-1}$ & $4.8693 \times 10^{-10}$ & $5.1637 \times 10^{-11}$ \\
1897 & 27 & $1.4780 \times 10^{-1}$ & $3.2077 \times 10^{-10}$ & $4.7410 \times 10^{-11}$ \\
1898 & 28 & $4.6064 \times 10^{-1}$ & $9.3109 \times 10^{-11}$ & $4.2890 \times 10^{-11}$ \\
1899 & 29 & $1.5262 \times 10^{-1}$ & $2.6852 \times 10^{-10}$ & $4.0982 \times 10^{-11}$ \\
1900 & 30 & $6.4868 \times 10^{-2}$ & $6.0223 \times 10^{-10}$ & $3.9066 \times 10^{-11}$ \\
1901 & 31 & $3.2751 \times 10^{-2}$ & $1.1357 \times 10^{-9}$ & $3.7196 \times 10^{-11}$ \\
1902 & 32 & $1.0198 \times 10^{-2}$ & $3.5265 \times 10^{-9}$ & $3.5961 \times 10^{-11}$ \\
1903 & 33 & $7.7135 \times 10^{-3}$ & $4.4168 \times 10^{-9}$ & $3.4069 \times 10^{-11}$ \\
1904 & 34 & $3.4940 \times 10^{-3}$ & $9.3872 \times 10^{-9}$ & $3.2799 \times 10^{-11}$ \\
1905 & 35 & $1.1907 \times 10^{-3}$ & $2.6811 \times 10^{-8}$ & $3.1924 \times 10^{-11}$ \\
\hline
\end{tabular}

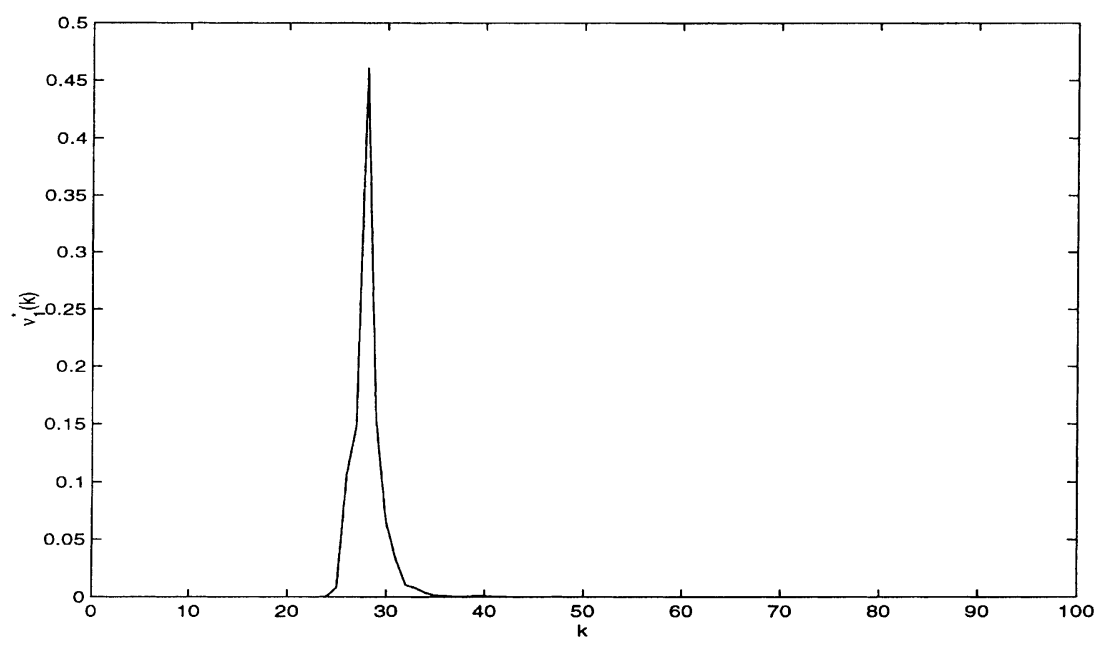

Fig. 1. The posterior probability $v_{1}^{*}(k \mid \underline{x})$ of the change-point $k$ in Nile data. 
The posterior probability $v_{1}^{*}(k \mid \underline{x})$ is maximized at $k=28$ corresponding to the conditional $p$-value : $p_{\lambda=1}(28)=9.3109 \times 10^{-11}$ and to the unconditional $p$ value $\sum_{k=1}^{100} v_{1}^{*}(k \mid \underline{x}) p_{\lambda=1}(k)=1.2785 \times 10^{-9}$.

The other posterior probability $v_{1}^{*}(k \mid \underline{x})$ is below $10^{-4}$ and then is omitted. The 95\% HPD credible set is $\{26,27,28,29,30,31\}$. For priors $v_{2}$ and $v_{3}$, the results are similar and are given in Appendix A (see Tables 4 and 5).

\subsection{The Poisson case}

In this section, we apply the previous results to the data used by Henderson and Matthews [7] and West and Ogden [13]. The data represent the number of haemolytic uraemic syndrome (HUS) cases in Birmingham and Newcastle from 1970 to 1989.

Henderson and Matthews [7] propose the following Poisson change-point model

$$
X_{i} \mid \theta_{1}, \theta_{2}, k \sim \begin{cases}\mathscr{P}\left(\theta_{1}\right) & \text { if } i=1, \ldots, k \\ \mathscr{P}\left(\theta_{2}\right) & \text { if } i=k+1, \ldots, n\end{cases}
$$

where $X_{i}$ denotes the number of HUS cases for the year $i$ and $\mathscr{P}(\theta)$ is the Poisson distribution with mean $\theta$. Henderson and Matthews [7] showed for the at most one change-point case that the change occurs at $k=11$ for the Birmingham data and $k=15$ for the Newcastle data. They used the loglikelihood statistic given as the change occurs and the resulting test $\left(\chi^{2}\right.$-test) is significant. West and Ogden [13] supposed that data over $T$ unit time periods, $X_{1}, \ldots, X_{T}$, are observed where $X_{i}$ represents the number of events that occurred in the $i$ th time period. Then they used a Poisson process to suppress the hypothesis that the change is an integer. They used the following model:

$$
X_{i} \mid \theta_{1}, \theta_{2}, \tau \sim \begin{cases}\mathscr{P}\left(\theta_{1}\right) & \text { if } i=1, \ldots,[\tau] \\ \mathscr{P}\left((\tau-[\tau]) \theta_{1}+(1-\tau+[\tau]) \theta_{2}\right) & \text { if } i=[\tau]+1 \\ \mathscr{P}\left(\theta_{2}\right) & \text { if } i=[\tau]+2, \ldots, T\end{cases}
$$

where $[x]$ denotes the greatest integer function of $x$.

They used only the Newcastle data and obtained (maximum-likelihood method), the value $\tau=14.944$ which closely corresponds with the estimate of 15 given by Henderson and Matthews [7]. The Birmingham data for the $v_{1}$ prior distribution are given in Table 2 (see also Fig. 2).

The posterior probability $v_{1}^{*}(k \mid \underline{x})$ is maximized at $k=11$ corresponding to the conditional $p$-value $p_{\lambda=1}(11)<10^{-15}$ and to the unconditional $p$-value $\sum_{k=1}^{20} v_{1}^{*}(k \mid \underline{x}) p_{\lambda=1}(k)<10^{-13}$.

The $98 \%$ HPD credible set is $\{11\}$. We obtain the same results as in [7]. 
Table 2

The posterior probability $v_{1}^{*}(k \mid \underline{x})$ of the change-point $k$ in Birmingham data

\begin{tabular}{lllll}
\hline Year & $k$ & $v_{1}^{*}(k \mid \underline{x})$ & $p_{\lambda=1}(k)$ & $v_{1}^{*}(k \mid \underline{x}) p_{\lambda=1}(k)$ \\
\hline 1970 & 1 & $1.4876 \times 10^{-11}$ & $4.7390 \times 10^{-4}$ & $7.0498 \times 10^{-15}$ \\
1971 & 2 & $3.3047 \times 10^{-13}$ & $6.7317 \times 10^{-3}$ & $2.2246 \times 10^{-15}$ \\
1972 & 3 & $2.9220 \times 10^{-13}$ & $3.1026 \times 10^{-3}$ & $9.0655 \times 10^{-16}$ \\
1973 & 4 & $9.0299 \times 10^{-13}$ & $4.7893 \times 10^{-4}$ & $4.3246 \times 10^{-16}$ \\
1974 & 5 & $3.7449 \times 10^{-12}$ & $6.3896 \times 10^{-5}$ & $2.3928 \times 10^{-16}$ \\
1975 & 6 & $6.2817 \times 10^{-11}$ & $2.2273 \times 10^{-6}$ & $1.3991 \times 10^{-16}$ \\
1976 & 7 & $6.3035 \times 10^{-9}$ & $1.3591 \times 10^{-8}$ & $8.5672 \times 10^{-17}$ \\
1977 & 8 & $1.3855 \times 10^{-6}$ & $4.0603 \times 10^{-11}$ & $5.6256 \times 10^{-17}$ \\
1978 & 9 & $2.4337 \times 10^{-5}$ & $1.6864 \times 10^{-12}$ & $4.1043 \times 10^{-17}$ \\
1979 & 10 & $3.4866 \times 10^{-3}$ & $8.6597 \times 10^{-15}$ & $3.0193 \times 10^{-17}$ \\
1980 & 11 & $9.8159 \times 10^{-1}$ & $<10^{-15}$ & $<10^{-15}$ \\
1981 & 12 & $1.4867 \times 10^{-2}$ & $1.3323 \times 10^{-15}$ & $1.9807 \times 10^{-17}$ \\
1982 & 13 & $2.6371 \times 10^{-6}$ & $7.5218 \times 10^{-12}$ & $1.9836 \times 10^{-17}$ \\
1983 & 14 & $1.9022 \times 10^{-5}$ & $8.7619 \times 10^{-13}$ & $1.6667 \times 10^{-17}$ \\
1984 & 15 & $4.2751 \times 10^{-6}$ & $3.5805 \times 10^{-12}$ & $1.5307 \times 10^{-17}$ \\
1985 & 16 & $4.1136 \times 10^{-8}$ & $3.7801 \times 10^{-10}$ & $1.5550 \times 10^{-17}$ \\
1986 & 17 & $1.9867 \times 10^{-12}$ & $1.0500 \times 10^{-5}$ & $2.0859 \times 10^{-17}$ \\
1987 & 18 & $1.8073 \times 10^{-15}$ & $2.0634 \times 10^{-2}$ & $3.7291 \times 10^{-17}$ \\
1988 & 19 & $4.8881 \times 10^{-16}$ & $1.0720 \times 10^{-1}$ & $5.2400 \times 10^{-17}$ \\
1989 & 20 & $1.2913 \times 10^{-13}$ & $9.9505 \times 10^{-3}$ & $1.2849 \times 10^{-15}$ \\
\hline
\end{tabular}

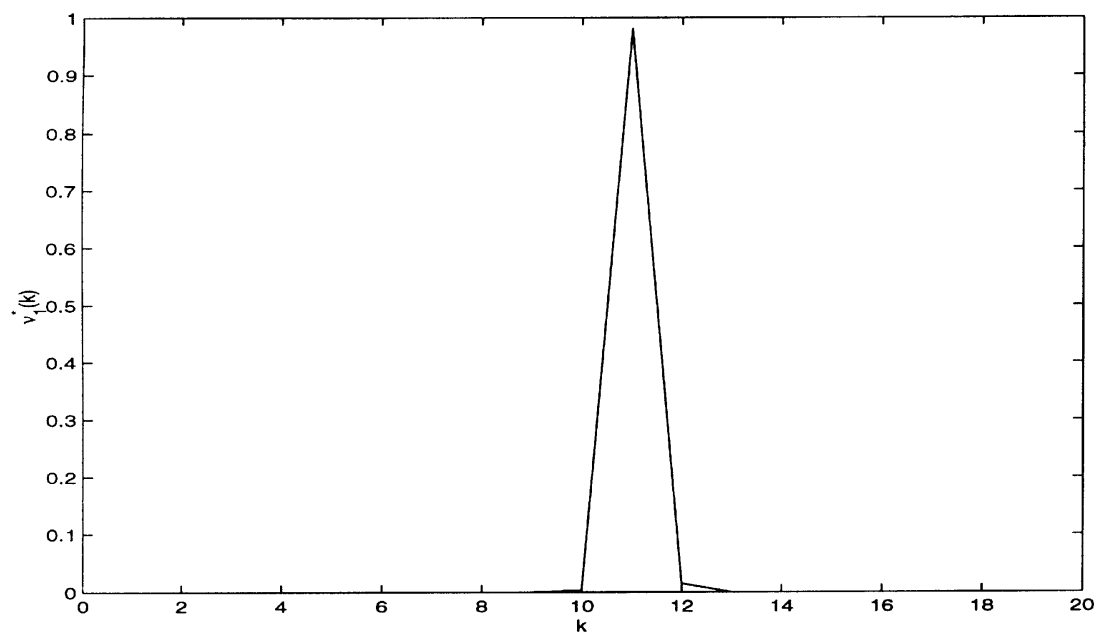

Fig. 2. The posterior probability $v_{1}^{*}(k \mid \underline{x})$ of the change-point $k$ in Birmingham data.

For priors $v_{2}$ and $v_{3}$, the results are similar and are given in Appendix A (see Tables 6 and 7).

The Newcastle data for the $v_{1}$ prior distribution are presented in Table 3 (see also Fig. 3). 
Table 3

The posterior probability $v_{1}^{*}(k \mid \underline{x})$ of the change-point $k$ in Newcastle data

\begin{tabular}{lllll}
\hline Year & $k$ & $v_{1}^{*}(k \mid \underline{x})$ & $p_{\lambda=1}(k)$ & $v_{1}^{*}(k \mid \underline{x}) p_{\lambda=1}(k)$ \\
\hline 1970 & 1 & $2.3497 \times 10^{-10}$ & $3.5570 \times 10^{-1}$ & $8.3580 \times 10^{-11}$ \\
1971 & 2 & $2.8257 \times 10^{-10}$ & $5.4046 \times 10^{-2}$ & $1.5271 \times 10^{-11}$ \\
1972 & 3 & $2.2793 \times 10^{-9}$ & $2.1065 \times 10^{-3}$ & $4.8015 \times 10^{-12}$ \\
1973 & 4 & $4.9441 \times 10^{-8}$ & $4.2230 \times 10^{-5}$ & $2.0879 \times 10^{-12}$ \\
1974 & 5 & $1.3692 \times 10^{-7}$ & $8.7600 \times 10^{-6}$ & $1.1994 \times 10^{-12}$ \\
1975 & 6 & $7.9344 \times 10^{-6}$ & $8.7104 \times 10^{-8}$ & $6.9112 \times 10^{-13}$ \\
1976 & 7 & $1.7190 \times 10^{-4}$ & $2.6161 \times 10^{-9}$ & $4.4969 \times 10^{-13}$ \\
1977 & 8 & $3.3230 \times 10^{-7}$ & $1.1952 \times 10^{-6}$ & $3.9715 \times 10^{-13}$ \\
1978 & 9 & $2.3912 \times 10^{-7}$ & $1.2942 \times 10^{-6}$ & $3.0947 \times 10^{-13}$ \\
1979 & 10 & $6.2282 \times 10^{-6}$ & $3.5521 \times 10^{-8}$ & $2.2123 \times 10^{-13}$ \\
1980 & 11 & $6.8085 \times 10^{-6}$ & $2.6499 \times 10^{-8}$ & $1.8042 \times 10^{-13}$ \\
1981 & 12 & $1.4901 \times 10^{-3}$ & $8.8467 \times 10^{-11}$ & $1.3183 \times 10^{-13}$ \\
1982 & 13 & $3.0148 \times 10^{-3}$ & $3.6818 \times 10^{-11}$ & $1.1100 \times 10^{-13}$ \\
1983 & 14 & $3.8235 \times 10^{-2}$ & $2.4041 \times 10^{-12}$ & $9.1919 \times 10^{-14}$ \\
1984 & 15 & $9.5703 \times 10^{-1}$ & $8.0380 \times 10^{-14}$ & $7.6926 \times 10^{-14}$ \\
1985 & 16 & $4.1173 \times 10^{-5}$ & $2.1319 \times 10^{-9}$ & $8.7777 \times 10^{-14}$ \\
1986 & 17 & $6.3489 \times 10^{-9}$ & $1.8199 \times 10^{-5}$ & $1.1554 \times 10^{-13}$ \\
1987 & 18 & $1.0839 \times 10^{-9}$ & $1.1593 \times 10^{-4}$ & $1.2566 \times 10^{-13}$ \\
1988 & 19 & $1.1139 \times 10^{-10}$ & $1.4078 \times 10^{-3}$ & $1.5682 \times 10^{-13}$ \\
1989 & 20 & $4.0572 \times 10^{-10}$ & $1.8217 \times 10^{-2}$ & $7.3912 \times 10^{-12}$ \\
\hline & & & & \\
\hline
\end{tabular}

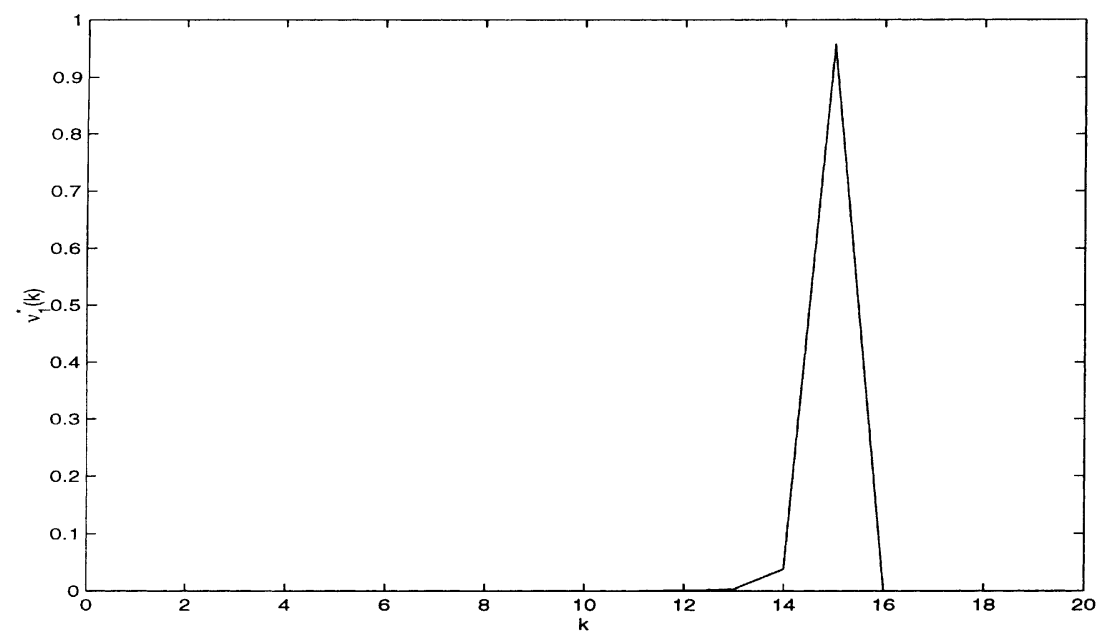

Fig. 3. The posterior probability $v_{1}^{*}(k \mid \underline{x})$ of the change-point $k$ in Newcastle data.

The posterior probability $v_{1}^{*}(k \mid \underline{x})$ is maximized at $k=15$ corresponding to the conditional $p$-value $p_{\lambda=1}(15)<10^{-13}$ and to the unconditional $p$-value $\sum_{k=1}^{20} v_{1}^{*}(k \mid \underline{x}) p_{\lambda=1}(k)<10^{-9}$. The $95 \%$ HPD credible set is $\{15\}$. 
For priors $v_{2}$ and $v_{3}$, the results are similar and are given in Appendix A (see Tables 8 and 9).

\section{Appendix A}

The posterior probability $v_{2}^{*}(k \mid \underline{x})$ of the change-point $k$ in Nile data is given in Table 4.

The posterior probability $v_{2}^{*}(k \mid \underline{x})$ is maximized at $k=28$ and corresponding to the conditional $p$-value $p_{\lambda=1}(28)=9.3109 \times 10^{-11}$ and to the unconditional $p$-value $\sum_{k=1}^{100} v_{2}^{*}(k \mid \underline{x}) p_{\lambda=1}(k)=1.4232 \times 10^{-9}$.

The other posterior probability $v_{2}^{*}(k \mid \underline{x})$ is below $10^{-4}$ and is omitted. The $95 \%$ HPD credible set is $\{26,27,28,29,30,31\}$.

The posterior probability $v_{3}^{*}(k \mid \underline{x})$ of the change-point $k$ in Nile data is given in Table 5.

Table 4

The posterior probability $v_{2}^{*}(k \mid \underline{x})$ of the change-point $k$ in Nile data

\begin{tabular}{lllll}
\hline Year & $k$ & $v_{2}^{*}(k \mid \underline{x})$ & $p_{\lambda=1}(k)$ & $v_{2}^{*}(k \mid \underline{x}) p_{\lambda=1}(k)$ \\
\hline 1895 & 25 & $7.9807 \times 10^{-3}$ & $7.3382 \times 10^{-9}$ & $5.8563 \times 10^{-11}$ \\
1896 & 26 & $1.0431 \times 10^{-1}$ & $4.8693 \times 10^{-10}$ & $5.0790 \times 10^{-11}$ \\
1897 & 27 & $1.4651 \times 10^{-1}$ & $3.2077 \times 10^{-10}$ & $4.6997 \times 10^{-11}$ \\
1898 & 28 & $4.6013 \times 10^{-1}$ & $9.3109 \times 10^{-11}$ & $4.2843 \times 10^{-11}$ \\
1899 & 29 & $1.5361 \times 10^{-1}$ & $2.6852 \times 10^{-10}$ & $4.1248 \times 10^{-11}$ \\
1900 & 30 & $6.5780 \times 10^{-2}$ & $6.0223 \times 10^{-10}$ & $3.9615 \times 10^{-11}$ \\
1901 & 31 & $3.3458 \times 10^{-2}$ & $1.1357 \times 10^{-9}$ & $3.7999 \times 10^{-11}$ \\
1902 & 32 & $1.0495 \times 10^{-2}$ & $3.5265 \times 10^{-9}$ & $3.7009 \times 10^{-11}$ \\
1903 & 33 & $7.9950 \times 10^{-3}$ & $4.4168 \times 10^{-9}$ & $3.5312 \times 10^{-11}$ \\
1904 & 34 & $3.6484 \times 10^{-3}$ & $9.3872 \times 10^{-9}$ & $3.4249 \times 10^{-11}$ \\
1905 & 35 & $1.2523 \times 10^{-3}$ & $2.6811 \times 10^{-8}$ & $3.3576 \times 10^{-11}$ \\
\hline
\end{tabular}

Table 5

The posterior probability $v_{3}^{*}(k \mid \underline{x})$ of the change-point $k$ in Nile data

\begin{tabular}{lllll}
\hline Year & $k$ & $v_{3}^{*}(k \mid \underline{x})$ & $p_{\lambda=1}(k)$ & $v_{3}^{*}(k \mid \underline{x}) p_{\lambda=1}(k)$ \\
\hline 1895 & 25 & $6.4593 \times 10^{-3}$ & $7.3382 \times 10^{-9}$ & $4.7399 \times 10^{-11}$ \\
1896 & 26 & $9.0458 \times 10^{-2}$ & $4.8693 \times 10^{-10}$ & $4.4047 \times 10^{-11}$ \\
1897 & 27 & $1.3578 \times 10^{-1}$ & $3.2077 \times 10^{-10}$ & $4.3553 \times 10^{-11}$ \\
1898 & 28 & $4.5450 \times 10^{-1}$ & $9.3109 \times 10^{-11}$ & $4.2318 \times 10^{-11}$ \\
1899 & 29 & $1.6134 \times 10^{-1}$ & $2.6852 \times 10^{-10}$ & $4.3324 \times 10^{-11}$ \\
1900 & 30 & $7.3305 \times 10^{-2}$ & $6.0223 \times 10^{-10}$ & $4.4147 \times 10^{-11}$ \\
1901 & 31 & $3.9478 \times 10^{-2}$ & $1.1357 \times 10^{-9}$ & $4.4835 \times 10^{-11}$ \\
1902 & 32 & $1.3085 \times 10^{-2}$ & $3.5265 \times 10^{-9}$ & $4.6144 \times 10^{-11}$ \\
1903 & 33 & $1.0516 \times 10^{-2}$ & $4.4168 \times 10^{-9}$ & $4.6448 \times 10^{-11}$ \\
1904 & 34 & $5.0523 \times 10^{-3}$ & $9.3872 \times 10^{-9}$ & $4.7426 \times 10^{-11}$ \\
1905 & 35 & $1.8230 \times 10^{-3}$ & $2.6811 \times 10^{-8}$ & $4.8877 \times 10^{-11}$ \\
\hline
\end{tabular}


The posterior probability $v_{3}^{*}(k \mid \underline{x})$ is maximized at $k=28$ and corresponding to the conditional $p$-value $p_{\lambda=1}(28)=9.310910^{-11}$ and to the unconditional $p$ value $\sum_{k=1}^{100} v_{3}^{*}(k \mid \underline{x}) p_{\lambda=1}(k)=3.2503 \times 10^{-9}$. The other posterior probability $v_{3}^{*}(k \mid \underline{x})$ is below $10^{-4}$ and is omitted. The 95\% HPD credible set is $\{26,27,28,29,30,31\}$.

The Birmingham data for the $v_{2}$ prior distribution are given in Table 6 .

The posterior probability $v_{2}^{*}(k \mid \underline{x})$ is maximized at $k=11$ and corresponding to the conditional $p$-value $p_{\lambda=1}(11)<10^{-15}$ and to the unconditional $p$-value $\sum_{k=1}^{20} v_{2}^{*}(k \mid \underline{x}) p_{\lambda=1}(k)<10^{-13}$. The $98 \%$ HPD credible set is $\{11\}$.

The Birmingham data for the $v_{3}$ prior distribution are given in Table 7.

The posterior probability $v_{3}^{*}(k \mid \underline{x})$ is maximized at $k=11$ and corresponding to the conditional $p$-value $p_{\lambda=1}(11)<10^{-15}$ and to the unconditional $p$-value $\sum_{k=1}^{20} v_{3}^{*}(k \mid \underline{x}) p_{\lambda=1}(k)<10^{-13}$. The $97 \%$ HPD credible set is $\{11\}$.

The Newcastle data for the $v_{2}$ prior distribution are given in Table 8 .

The posterior probability $v_{2}^{*}(k \mid \underline{x})$ is maximized at $k=15$ and corresponding to the conditional $p$-value $p_{\lambda=1}(15)<10^{-13}$ and to the unconditional $p$-value $\sum_{k=1}^{20} v_{2}^{*}(k \mid \underline{x}) p_{\lambda=1}(k)<10^{-9}$. The $95 \%$ HPD credible set is $\{15\}$.

The Newcastle data for the $v_{3}$ prior distribution are given in Table 9.

Table 6

The posterior probability $v_{2}^{*}(k \mid \underline{x})$ of the change-point $k$ in Birmingham data

\begin{tabular}{lllll}
\hline Year & $k$ & $v_{2}^{*}(k \mid \underline{x})$ & $p_{\lambda=1}(k)$ & $v_{2}^{*}(k \mid \underline{x}) p_{\lambda=1}(k)$ \\
\hline 1970 & 1 & $4.4244 \times 10^{-12}$ & $4.7390 \times 10^{-4}$ & $2.0967 \times 10^{-15}$ \\
1971 & 2 & $1.5032 \times 10^{-13}$ & $6.7317 \times 10^{-3}$ & $1.0119 \times 10^{-15}$ \\
1972 & 3 & $1.6322 \times 10^{-13}$ & $3.1026 \times 10^{-3}$ & $5.0641 \times 10^{-16}$ \\
1973 & 4 & $5.7517 \times 10^{-13}$ & $4.7893 \times 10^{-4}$ & $2.7546 \times 10^{-16}$ \\
1974 & 5 & $2.6281 \times 10^{-12}$ & $6.3896 \times 10^{-5}$ & $1.6792 \times 10^{-16}$ \\
1975 & 6 & $4.7667 \times 10^{-11}$ & $2.2273 \times 10^{-6}$ & $1.0617 \times 10^{-16}$ \\
1976 & 7 & $5.1129 \times 10^{-9}$ & $1.3591 \times 10^{-8}$ & $6.9491 \times 10^{-17}$ \\
1977 & 8 & $1.1922 \times 10^{-6}$ & $4.0603 \times 10^{-11}$ & $4.8408 \times 10^{-17}$ \\
1978 & 9 & $2.2096 \times 10^{-5}$ & $1.6864 \times 10^{-12}$ & $3.7263 \times 10^{-17}$ \\
1979 & 10 & $3.3266 \times 10^{-3}$ & $8.6597 \times 10^{-15}$ & $2.8807 \times 10^{-17}$ \\
1980 & 11 & $9.8109 \times 10^{-1}$ & $<10^{-15}$ & $<10^{-15}$ \\
1981 & 12 & $1.5526 \times 10^{-2}$ & $1.3323 \times 10^{-15}$ & $2.0685 \times 10^{-17}$ \\
1982 & 13 & $2.8714 \times 10^{-6}$ & $7.5218 \times 10^{-12}$ & $2.1598 \times 10^{-17}$ \\
1983 & 14 & $2.1554 \times 10^{-5}$ & $8.7619 \times 10^{-13}$ & $1.8885 \times 10^{-17}$ \\
1984 & 15 & $5.0328 \times 10^{-6}$ & $3.5805 \times 10^{-12}$ & $1.8020 \times 10^{-17}$ \\
1985 & 16 & $5.0240 \times 10^{-8}$ & $3.7801 \times 10^{-10}$ & $1.8991 \times 10^{-17}$ \\
1986 & 17 & $2.5138 \times 10^{-12}$ & $1.0500 \times 10^{-5}$ & $2.6395 \times 10^{-17}$ \\
1987 & 18 & $2.3665 \times 10^{-15}$ & $2.0634 \times 10^{-2}$ & $4.8829 \times 10^{-17}$ \\
1988 & 19 & $6.6160 \times 10^{-16}$ & $1.0720 \times 10^{-1}$ & $7.0923 \times 10^{-17}$ \\
1989 & 20 & $1.1263 \times 10^{-12}$ & $9.9505 \times 10^{-3}$ & $1.1208 \times 10^{-14}$ \\
\hline & & & &
\end{tabular}


Table 7

The posterior probability $v_{3}^{*}(k \mid \underline{x})$ of the change-point $k$ in Birmingham data

\begin{tabular}{lllll}
\hline Year & $k$ & $v_{3}^{*}(k \mid \underline{x})$ & $p_{\lambda=1}(k)$ & $v_{3}^{*}(k \mid \underline{x}) p_{\lambda=1}(k)$ \\
\hline 1970 & 1 & $2.2492 \times 10^{-13}$ & $4.7390 \times 10^{-4}$ & $1.0659 \times 10^{-16}$ \\
1971 & 2 & $1.4989 \times 10^{-14}$ & $6.7317 \times 10^{-3}$ & $1.0090 \times 10^{-16}$ \\
1972 & 3 & $2.6507 \times 10^{-14}$ & $3.1026 \times 10^{-3}$ & $8.2239 \times 10^{-17}$ \\
1973 & 4 & $1.3653 \times 10^{-13}$ & $4.7893 \times 10^{-4}$ & $6.5386 \times 10^{-17}$ \\
1974 & 5 & $8.4930 \times 10^{-13}$ & $6.3896 \times 10^{-5}$ & $5.4266 \times 10^{-17}$ \\
1975 & 6 & $1.9945 \times 10^{-11}$ & $2.2273 \times 10^{-6}$ & $4.4423 \times 10^{-17}$ \\
1976 & 7 & $2.6685 \times 10^{-9}$ & $1.3591 \times 10^{-8}$ & $3.6268 \times 10^{-17}$ \\
1977 & 8 & $7.5414 \times 10^{-7}$ & $4.0603 \times 10^{-11}$ & $3.0620 \times 10^{-17}$ \\
1978 & 9 & $1.6558 \times 10^{-5}$ & $1.6864 \times 10^{-12}$ & $2.7924 \times 10^{-17}$ \\
1979 & 10 & $2.8993 \times 10^{-3}$ & $8.6597 \times 10^{-15}$ & $2.5107 \times 10^{-17}$ \\
1980 & 11 & $9.7951 \times 10^{-1}$ & $<10^{-15}$ & $<10^{-15}$ \\
1981 & 12 & $1.7533 \times 10^{-2}$ & $1.3323 \times 10^{-15}$ & $2.3358 \times 10^{-17}$ \\
1982 & 13 & $3.6283 \times 10^{-6}$ & $7.5218 \times 10^{-12}$ & $2.7291 \times 10^{-17}$ \\
1983 & 14 & $3.0198 \times 10^{-5}$ & $8.7619 \times 10^{-13}$ & $2.6459 \times 10^{-17}$ \\
1984 & 15 & $7.7564 \times 10^{-6}$ & $3.5805 \times 10^{-12}$ & $2.7772 \times 10^{-17}$ \\
1985 & 16 & $8.4586 \times 10^{-8}$ & $3.7801 \times 10^{-10}$ & $3.1974 \times 10^{-17}$ \\
1986 & 17 & $4.5957 \times 10^{-12}$ & $1.0500 \times 10^{-5}$ & $4.8253 \times 10^{-17}$ \\
1987 & 18 & $4.6726 \times 10^{-15}$ & $2.0634 \times 10^{-2}$ & $9.6413 \times 10^{-17}$ \\
1988 & 19 & $1.4042 \times 10^{-15}$ & $1.0720 \times 10^{-1}$ & $1.5053 \times 10^{-16}$ \\
1989 & 20 & $3.7094 \times 10^{-13}$ & $9.9505 \times 10^{-3}$ & $3.6910 \times 10^{-15}$ \\
\hline
\end{tabular}

Table 8

The posterior probability $v_{2}^{*}(k \mid \underline{x})$ of the change-point $k$ in Newcastle data

\begin{tabular}{lllll}
\hline Year & $k$ & $v_{2}^{*}(k \mid \underline{x})$ & $p_{\lambda=1}(k)$ & $v_{2}^{*}(k \mid \underline{x}) p_{\lambda=1}(k)$ \\
\hline 1970 & 1 & $2.3497 \times 10^{-10}$ & $3.5570 \times 10^{-1}$ & $8.3580 \times 10^{-11}$ \\
1971 & 2 & $2.8257 \times 10^{-10}$ & $5.4046 \times 10^{-2}$ & $1.5271 \times 10^{-11}$ \\
1972 & 3 & $2.2793 \times 10^{-9}$ & $2.1065 \times 10^{-3}$ & $4.8015 \times 10^{-12}$ \\
1973 & 4 & $4.9441 \times 10^{-8}$ & $4.2230 \times 10^{-5}$ & $2.0879 \times 10^{-12}$ \\
1974 & 5 & $1.3692 \times 10^{-7}$ & $8.7600 \times 10^{-6}$ & $1.1994 \times 10^{-12}$ \\
1975 & 6 & $7.9344 \times 10^{-6}$ & $8.7104 \times 10^{-8}$ & $6.9112 \times 10^{-13}$ \\
1976 & 7 & $1.7190 \times 10^{-4}$ & $2.6161 \times 10^{-9}$ & $4.4969 \times 10^{-13}$ \\
1977 & 8 & $3.3230 \times 10^{-7}$ & $1.1952 \times 10^{-6}$ & $3.9715 \times 10^{-13}$ \\
1978 & 9 & $2.3912 \times 10^{-7}$ & $1.2942 \times 10^{-6}$ & $3.0947 \times 10^{-13}$ \\
1979 & 10 & $6.2282 \times 10^{-6}$ & $3.5521 \times 10^{-8}$ & $2.2123 \times 10^{-13}$ \\
1980 & 11 & $6.8085 \times 10^{-6}$ & $2.6499 \times 10^{-8}$ & $1.8042 \times 10^{-13}$ \\
1981 & 12 & $1.4901 \times 10^{-3}$ & $8.8467 \times 10^{-11}$ & $1.3183 \times 10^{-13}$ \\
1982 & 13 & $3.0148 \times 10^{-3}$ & $3.6818 \times 10^{-11}$ & $1.1100 \times 10^{-13}$ \\
1983 & 14 & $3.8235 \times 10^{-2}$ & $2.4041 \times 10^{-12}$ & $9.1919 \times 10^{-14}$ \\
1984 & 15 & $9.5703 \times 10^{-1}$ & $8.0380 \times 10^{-14}$ & $7.6926 \times 10^{-14}$ \\
1985 & 16 & $4.1173 \times 10^{-5}$ & $2.1319 \times 10^{-9}$ & $8.7777 \times 10^{-14}$ \\
1986 & 17 & $6.3489 \times 10^{-9}$ & $1.8199 \times 10^{-5}$ & $1.1554 \times 10^{-13}$ \\
1987 & 18 & $1.0839 \times 10^{-9}$ & $1.1593 \times 10^{-4}$ & $1.2566 \times 10^{-13}$ \\
1988 & 19 & $1.1139 \times 10^{-10}$ & $1.4078 \times 10^{-3}$ & $1.5682 \times 10^{-13}$ \\
1989 & 20 & $4.0572 \times 10^{-10}$ & $1.8217 \times 10^{-2}$ & $7.3912 \times 10^{-12}$ \\
\hline
\end{tabular}


Table 9

The posterior probability $v_{3}^{*}(k \mid \underline{x})$ of the change-point $k$ in Newcastle data

\begin{tabular}{lllll}
\hline Year & $k$ & $v_{3}^{*}(k \mid \underline{x})$ & $p_{\lambda=1}(k)$ & $v_{3}^{*}(k \mid \underline{x}) p_{\lambda=1}(k)$ \\
\hline 1970 & 1 & $1.9703 \times 10^{-12}$ & $3.5570 \times 10^{-1}$ & $7.0082 \times 10^{-13}$ \\
1971 & 2 & $7.1080 \times 10^{-12}$ & $5.4046 \times 10^{-2}$ & $3.8416 \times 10^{-13}$ \\
1972 & 3 & $1.1467 \times 10^{-10}$ & $2.1065 \times 10^{-3}$ & $2.4156 \times 10^{-13}$ \\
1973 & 4 & $4.1457 \times 10^{-9}$ & $4.2230 \times 10^{-5}$ & $1.7507 \times 10^{-13}$ \\
1974 & 5 & $1.7221 \times 10^{-8}$ & $8.7600 \times 10^{-6}$ & $1.5086 \times 10^{-13}$ \\
1975 & 6 & $1.3971 \times 10^{-6}$ & $8.7104 \times 10^{-8}$ & $1.2170 \times 10^{-13}$ \\
1976 & 7 & $4.0358 \times 10^{-5}$ & $2.6161 \times 10^{-9}$ & $1.0558 \times 10^{-13}$ \\
1977 & 8 & $1.0031 \times 10^{-7}$ & $1.1952 \times 10^{-6}$ & $1.1989 \times 10^{-13}$ \\
1978 & 9 & $9.0227 \times 10^{-8}$ & $1.2942 \times 10^{-6}$ & $1.1677 \times 10^{-13}$ \\
1979 & 10 & $2.8723 \times 10^{-6}$ & $3.5521 \times 10^{-8}$ & $1.0203 \times 10^{-13}$ \\
1980 & 11 & $3.7679 \times 10^{-6}$ & $2.6499 \times 10^{-8}$ & $9.9846 \times 10^{-14}$ \\
1981 & 12 & $9.7458 \times 10^{-4}$ & $8.8467 \times 10^{-11}$ & $8.6218 \times 10^{-14}$ \\
1982 & 13 & $2.3004 \times 10^{-3}$ & $3.6818 \times 10^{-11}$ & $8.4698 \times 10^{-14}$ \\
1983 & 14 & $3.3663 \times 10^{-2}$ & $2.4041 \times 10^{-12}$ & $8.0928 \times 10^{-14}$ \\
1984 & 15 & $9.6297 \times 10^{-1}$ & $8.0380 \times 10^{-14}$ & $7.7403 \times 10^{-14}$ \\
1985 & 16 & $4.6953 \times 10^{-5}$ & $2.1319 \times 10^{-9}$ & $1.0010 \times 10^{-13}$ \\
1986 & 17 & $8.1451 \times 10^{-9}$ & $1.8199 \times 10^{-5}$ & $1.4823 \times 10^{-13}$ \\
1987 & 18 & $1.5541 \times 10^{-9}$ & $1.1593 \times 10^{-4}$ & $1.8017 \times 10^{-13}$ \\
1988 & 19 & $1.7747 \times 10^{-10}$ & $1.4078 \times 10^{-3}$ & $2.4984 \times 10^{-13}$ \\
1989 & 20 & $6.4638 \times 10^{-10}$ & $1.8217 \times 10^{-2}$ & $1.1775 \times 10^{-11}$ \\
\hline
\end{tabular}

The posterior probability $v_{3}^{*}(k \mid \underline{x})$ is maximized at $k=15$ and corresponding to the conditional $p$-value $p_{\lambda=1}(15)<10^{-13}$ and to the unconditional $p$-value $\sum_{k=1}^{20} v_{3}^{*}(k \mid \underline{x}) p_{\lambda=1}(k)<10^{-10}$. The $96 \%$ HPD credible set is $\{15\}$.

\section{References}

[1] H. Chernoff, S. Zacks, Estimating the current mean of a normal distribution which is subjected to change in time, Ann. Math. Statist. 35 (1964) 999-1018.

[2] G.W. Cobb, The problem of the Nile: conditional solution to a change-point problem, Biometrika 65 (1978) 243-251.

[3] J. Diaz, Bayesian detection of a change of scale parameter in sequences of independent Gamma random variables, J. Econometrics 19 (1982) 23-29.

[4] D. Ghorbanzadeh, R. Lounes, A Bayes procedure for detecting change in exponential random observations, Student 2 (2) (1998).

[5] D. Ghorbanzadeh, Detection of random change-point in one-parameter exponential families, Appl. Math. Comput. 77 (2 \& 3) (1996) 167-177.

[6] D. Ghorbanzadeh, R. Lounes, Deux procédures de détection de rupture pour des observations poissonniennes groupées, Rev. de Statist. Appl. XLIV (3) (1996) 47-61.

[7] R. Henderson, J.N.S. Matthews, An investigation of changepoints in the annual number of cases of haemolytic uraemic syndrome, Appl. Statist. 42 (3) (1993) 461-471.

[8] A. Kander, S. Zacks, Test procedure for possible changes in parameters of statistical distributions occuring at unknown time points, Ann. Mat. Statist. 37 (1966) 1196-1210. 
[9] D. Kim, A Bayesian significance test of the stationarity of regression parameters, Biometrika 78 (1991) 667-675.

[10] C.B. Lee, Bayesian analysis of a change-point in exponential families with applications, Comp. Statist. Data. Anal. 27 (1998) 195-208.

[11] U. Menzefricke, A Bayesian analysis of a change in the precision of a sequence of independent normal random variables at an unknown time point, Appl. Statist. 30 (1981) 141-146.

[12] A.F.M. Smith, A Bayesian approach to inference about a change-point in a sequence of random variables, Biometrika 62 (1975) 407-416.

[13] R.W. West, R.T. Ogden, Continuous-time estimation of a change-point in a Poisson process, J. Statist. Comput. Simul. 56 (1997) 293-302. 